\title{
On sharp surface force model: Effect of sharpening coefficient
}

\author{
Kurian J. Vachaparambil ( $₫)$, Kristian Etienne Einarsrud \\ Department of Materials Science and Engineering, Norwegian University of Science and Technology, 7491 Trondheim, Norway
}

\begin{abstract}
Amongst the multitude of approaches available in literature to reduce spurious velocities in Volume of Fluid approach, the Sharp Surface Force (SSF) model is increasingly being used due to its relative ease to implement. The SSF approach relies on a user-defined parameter, the sharpening coefficient, which determines the extent of the smeared nature of interface used to determine the surface tension force. In this paper, we use the SSF model implemented in OpenFOAM ${ }^{\otimes}$ to investigate the effect of this sharpening coefficient on spurious velocities and accuracy of dynamic, i.e., capillary rise, and static bubble simulations. Results show that increasing the sharpening coefficient generally reduces the spurious velocities in both static and dynamic cases. Although static millimeter sized bubbles were simulated with the whole range of sharpening coefficients, sub-millimeter sized bubbles show nonphysical behavior for values larger than 0.3. The accuracy of the capillary rise simulations has been observed to change non-linearly with the sharpening coefficient. This work illustrates the importance of using an optimized value of the sharpening coefficient with respect to spurious velocities and accuracy of the simulation.

\author{
Keywords \\ OpenFOAM $^{\otimes}$ \\ spurious velocities \\ two-phase flows \\ Volume of Fluid \\ Article History \\ Received: 6 January 2020 \\ Revised: 6 March 2020 \\ Accepted: 7 March 2020 \\ Research Article \\ (c) The Author(s) 2020
}

\section{Introduction}

Modelling surface tension dominant multiphase flows is relevant in a multitude of industrial processes like lab-on-chip, atomization, and boiling. One of the main approach to capture the interface dynamics is the Volume of Fluid method which uses the advection of scalar volume fraction based on algebraic (interface compression) or geometric (piecewise linear interface calculation or PLIC) reconstruction algorithms in order to preserve the sharpness of interfacial region (Cifani et al., 2016). The VOF based solver available in OpenFOAM ${ }^{\circledR}$, interFoam, which generates an interface which is smeared over a few computational cells uses the interface compression method due to its relative simplicity (Deshpande et al., 2012).

In the VOF approach used in interFoam, the volume fraction field is used to determine curvature and corresponding surface tension force based on models like the widely used Continuum Surface Force (CSF) approach (Brackbill et al., 1992). Due to the smeared nature of the interface, the curvature and the pressure jump across the interface obtained from the simulations do not match the theoretical value which generates spurious velocities

$\bowtie$ kurian.j.vachaparambil@ntnu.no,kurian_jomy@hotmail.com
(Deshpande et al.,2012).These spurious velocities introduce nonphysical flows near the interface which may cause the bubble to numerically drift as well as alter the heat/mass transfer coefficients in supersaturation and temperature driven phase change processes (Samkhaniani and Ansari, 2016; Saufi et al., 2019; Vachaparambil and Einarsrud, 2020). The works by Popinet (2018) and Deshpande et al. (2012) have reviewed the various approaches reported to mitigate these effects, namely: improved curvature estimation, force balance between surface tension and pressure gradient (for static cases), time step constraint when surface tension is calculated explicitly and temporally implicit approach to estimate surface tension.

Amongst the approaches proposed, the Sharp Surface Force model, developed by Raeini et al. (2012), estimates surface tension based on a smoothed interface curvature and a sharpened interface region defined using a user defined sharpening coefficient $\left(C_{\text {sh }}\right)$. This model, which is relatively simple to implement compared to height function based approach (Pavuluri et al., 2018), has been shown to reduce spurious velocities in comparison to commonly used CSF model (Pavuluri et al., 2018; Vachaparambil and Einarsrud, 2019a). The SSF model, 
using $C_{\mathrm{sh}} \in[0,0.5]$, has been used to simulate dynamic cases like rising bubbles (Vachaparambil and Einarsrud, 2019a), microfluidic T-junction (Soh et al., 2016), microchannels (Pavuluri et al., 2018), capillary rise (Raeini et al., 2012; Vachaparambil and Einarsrud, 2019a), interfacial mass transfer (Maes and Soulaine, 2018), and bubble growth (Vachaparambil and Einarsrud, 2020) whereas when modelling static cases, like stationary millimeter sized bubble, $C_{\text {sh }}$ is set equal to 0.98 (Vachaparambil and Einarsrud, 2019a). The choice of the value $C_{\text {sh }}$ used in the simulations is often heuristic and to the best knowledge of the authors there has not been a systematic attempt to quantify the effect of this user-defined parameter.

In this paper, we investigate the effect of the sharpening coefficient used in the SSF model, as developed on OpenFOAM $^{\circledR} 6$ by Vachaparambil and Einarsrud (2019a), to model two dimensional dynamic cases like capillary rise and static cases like millimeter and sub-millimeter bubbles. All the simulations discussed in this work use the sharpening coefficient typically used in simulating practical flow scenarios, i.e., $0 \leqslant C_{\text {sh }} \leqslant 0.5$ (based on the values used in Raeini et al. (2012), Soh et al. (2016), Maes and Soulaine (2018), Pavuluri et al. (2018), Vachaparambil and Einarsrud (2019a), Vachaparambil and Einarsrud (2020)).

\section{Governing equations}

The volume fraction $\left(\alpha_{1}\right)$ used in VOF method is a scalar field that is zero in gas phase, unity in the liquid phase, and $0<\alpha_{1}<1$ at the interface. The interface dynamics is captured based on the advection of $\alpha_{1}$ as

$$
\frac{\partial \alpha_{1}}{\partial t}+\nabla \cdot\left(\boldsymbol{U} \alpha_{1}\right)+\nabla \cdot\left(\alpha_{1}\left(1-\alpha_{1}\right) \boldsymbol{U}_{\mathrm{r}}\right)=0
$$

where $\boldsymbol{U}$ is the velocity in both phases and the third term is the interface compression term that acts in the interfacial region to prevent excessive smearing using $\boldsymbol{U}_{\mathrm{r}}$ which is defined as

$$
\boldsymbol{U}_{\mathrm{r}}=C_{\alpha}\left|\frac{\phi}{\left|\boldsymbol{S}_{\mathrm{f}}\right|}\right| \boldsymbol{n}
$$

where $C_{\alpha}, \phi, \boldsymbol{S}_{\mathrm{f}}$, and $\boldsymbol{n}$ represent adjustable compression factor (set equal to unity as recommended by Greenshields (2019)), volumetric flux across the cell face, cell face surface area, and unit normal to interface respectively (Deshpande et al., 2012). The fluid properties like density $(\rho)$ and viscosity $(\mu)$ are calculated as

$$
\chi=\chi_{1} \alpha_{1}+\chi_{2} \alpha_{2} \text {, where } \chi \in[\rho, \mu]
$$

where $\alpha_{2}=1-\alpha_{1}$. The mass conservation of the incom- pressible phases is described using continuity equation as

$$
\nabla \cdot \boldsymbol{U}=0
$$

The momentum equation is written based on a modified pressure $\left(p_{\mathrm{rgh}}\right)$, defined as $p_{\mathrm{rgh}}=p-\rho \boldsymbol{g} \cdot \boldsymbol{x}$, as

$$
\begin{gathered}
\frac{\partial \rho \boldsymbol{U}}{\partial t}+\nabla \cdot \rho \boldsymbol{U} \boldsymbol{U}=-\nabla p_{\mathrm{rgh}}-\boldsymbol{g} \cdot \boldsymbol{x} \nabla \rho \\
+\nabla \cdot \mu\left(\nabla \boldsymbol{U}+\nabla \boldsymbol{U}^{\mathrm{T}}\right)+\boldsymbol{F}_{\mathrm{ST}}
\end{gathered}
$$

where $\boldsymbol{F}_{\mathrm{ST}}$ is the surface tension modelled based on SSF described in Vachaparambil and Einarsrud (2019a) and Raeini et al. (2012). Initially, a smoothed volume fraction is obtained using a three consecutive smoothing steps ( $i=1$, $2,3)$ as

$$
\alpha_{i+1}^{s}=C\left\langle\left\langle\alpha_{i}^{s}\right\rangle_{\mathrm{c} \rightarrow \mathrm{f}}\right\rangle_{\mathrm{f} \rightarrow \mathrm{c}}+(1-C) \alpha_{i}^{s}
$$

where $\alpha_{1}^{s}=\alpha_{1}, C$ is equal to 0.5 , and $\left\langle\alpha_{1}\right\rangle_{\mathrm{c} \rightarrow \mathrm{f}}$ represents the interpolation of $\alpha_{1}$ from cell center to face. The unit normal to the smoothed interface is calculated and corrected for the effects of contact angle, see Vachaparambil and Einarsrud (2019a). Subsequently an initial estimate of curvature is calculated as

$$
\kappa_{1}=-\nabla \cdot \frac{\nabla \alpha_{4}^{s}}{\left|\nabla \alpha_{4}^{s}\right|+\delta}
$$

where $\delta$, defined as $10^{-8} /\left(\frac{\sum_{N} V_{i}}{N}\right)^{1 / 3}$, is used to prevent denominator from becoming zero. The curvature is smoothed using a two step procedure $(i=1,2)$ as

$$
\kappa_{i+1}^{s}=2 \sqrt{A} \kappa_{1}+(1-2 \sqrt{A}) \frac{\left\langle\left\langle w \kappa_{i}^{s}\right\rangle_{\mathrm{c} \rightarrow \mathrm{f}}\right\rangle_{\mathrm{f} \rightarrow \mathrm{c}}}{\left\langle\langle w\rangle_{\mathrm{c} \rightarrow \mathrm{f}}\right\rangle_{\mathrm{f} \rightarrow \mathrm{c}}}
$$

where $\kappa_{1}^{s}=\kappa_{1}, \quad w=\sqrt{A+0.001}$, and $A=\min (1, \max$ $\left.\left(\alpha_{1}, 0\right)\right)\left(1-\min \left(1, \max \left(\alpha_{1}, 0\right)\right)\right)$. The final curvature is calculated as

$$
\kappa_{\text {final }}=\frac{\left\langle w \kappa_{3}^{s}\right\rangle_{\mathrm{c} \rightarrow \mathrm{f}}}{\langle w\rangle_{\mathrm{c} \rightarrow \mathrm{f}}}
$$

The surface tension is estimated based on $\boldsymbol{F}_{\mathrm{ST}}=\sigma \kappa_{\text {final }}$. $\nabla \alpha_{\text {sh }}$, where $\alpha_{\text {sh }}$ is

$$
\alpha_{\mathrm{sh}}=\frac{1}{1-C_{\mathrm{sh}}}\left[\min \left(\max \left(\alpha_{1}, \frac{C_{\mathrm{sh}}}{2}\right), 1-\frac{C_{\mathrm{sh}}}{2}\right)-\frac{C_{\mathrm{sh}}}{2}\right]
$$

where $C_{\text {sh }}$ is the sharpening coefficient which when equal to zero produces $\alpha_{\mathrm{sh}}$ that is equivalent to $\alpha_{1}$. Due to the coupled nature of Eq. (4) and Eq. (5), these equations are solved by Pressure-Implicit with Splitting of Operators (PISO) algorithm (Deshpande et al., 2012). PISO algorithm involves estimation of a predicted velocity that is used to 
calculate pressure, using pressure correction equation, which is used to update the velocity in an iterative manner (Deshpande et al., 2012). In order to reduce spurious velocities, the force balance between pressure gradient, surface tension, and gravitational force due to discretization is ensured by calculating the gradients at cell faces as described in Deshpande et al. (2012). However the iterative procedure used to solve $p_{\text {rgh }}$, i.e., the PISO algorithm, converges based on a user defined tolerance (Deshpande et al., 2012). This tolerance, required to calculate $p_{\text {rgh }}$, introduces a force imbalance between surface tension, gravitational force, and pressure gradient which can be reduced by setting a very low convergence criterion, like $10^{-20}$ used in Table 1, as recommended by Deshpande et al. (2012).

\section{Computational domain and solver settings}

The governing equations are discretized using first and second order methods in time and space respectively, see Vachaparambil and Einarsrud (2019a), and solved based on methods described in Table 1. Other numerical settings like the sub-cycling of volume fraction equation and momentum predictor, which are relevant in solving the governing equations, are set based on OpenFOAM ${ }^{\circledR}$ default settings/recommendations for simulating multiphase flows which has also been used in Vachaparambil and Einarsrud (2019a). The simulations are run with no under-relaxation factor and maximum time step is calculated as

$$
\text { and } \begin{aligned}
\Delta t & \leqslant \frac{1}{2}\left(C_{2} \tau_{\mu}+\sqrt{\left(C_{2} \tau_{\mu}\right)^{2}+4 C_{1} \tau_{\rho}^{2}}\right) \\
\text { at } \Delta t & \leqslant \max \left(C_{2} \tau_{\mu}, 10 C_{1} \tau_{\rho}\right)
\end{aligned}
$$

where $C_{1}=0.01, C_{2}=10, \tau_{\mu}=\mu_{\text {avg }} \Delta x / \sigma$, and $\tau_{\rho}=$ $\sqrt{\rho_{\text {avg }}(\Delta x)^{3} / \sigma} \cdot \mu_{\text {avg }}$ and $\rho_{\text {avg }}$ are defined as the average dynamic viscosity and density between the phases and $\Delta x$ is the mesh resolution used in the simulations (Deshpande et al., 2012; Vachaparambil and Einarsrud, 2019a).

The fluid properties used in the both capillary rise and stationary bubble simulations are $\rho_{1}=1000 \mathrm{~kg} / \mathrm{m}^{3}, \rho_{2}=$ $1 \mathrm{~kg} / \mathrm{m}^{3}, v_{1}=10^{-6} \mathrm{~m}^{2} / \mathrm{s}, v_{2}=1.48 \times 10^{-5} \mathrm{~m}^{2} / \mathrm{s}$, and $\sigma=0.07$ $\mathrm{N} / \mathrm{m}$. The capillary rise simulations use $|\boldsymbol{g}|$ equal to 10 $\mathrm{m} / \mathrm{s}^{2}$ whereas stationary bubble simulations neglect gravity (Yamamoto et al., 2017; Vachaparambil and Einarsrud, 2019a).

The computational domain used for the capillary rise simulations is $20 \mathrm{~mm} \times 1 \mathrm{~mm}$ and meshed with a hexahedral grid of $400 \times 20$. This mesh is chosen based on the work by Yamamoto et al. (2017) that investigated the
Table 1 Solvers used for the discretized equation (Greenshields,

\begin{tabular}{|c|c|c|c|}
\hline Equation & Linear solver & Smoother/preconditioner & Tolerance \\
\hline $\begin{array}{l}\text { Pressure correction } \\
\text { equation }\end{array}$ & PCG & GAMG & $10^{-20}$ \\
\hline Momentum equation & smoothSolver & symGaussSeidel & $10^{-10}$ \\
\hline $\begin{array}{c}\text { Volume fraction } \\
\text { equation }\end{array}$ & smoothSolver & symGaussSeidel & $10^{-10}$ \\
\hline
\end{tabular}
2019)

effect of grid resolution on the accuracy of the capillary rise simulations. The boundary conditions used for $\alpha_{1}$ is zero gradient at the outlet, Dirichlet condition equal to one at inlet, and zero gradient with a constant contact angle of $45^{\circ}$ at the side boundaries. The modified pressure $\left(p_{\text {rgh }}\right)$ uses Dirichlet condition, equal to zero, at inlet and outlet but the side walls are assigned the zero gradient condition. The boundary conditions for $\boldsymbol{U}$ at side boundaries are set as no slip whereas the inlet and outlet are assigned a pressureinlet outlet velocity condition (Greenshields, 2019). The simulations are initialized with liquid column at a height of $8 \mathrm{~mm}$ (from the inlet) in the computational domain. These simulations are run until $1.5 \mathrm{~s}$ which is enough to reach steady capillary rise height with maximum time step, calculated based on Eq. (11), equal to $3.5 \mu$ s.

In order to model a stationary bubble of radius $R$, which is initialized at the center of a square computational domain of dimensions $4 R \times 4 R$, gravity is neglected. The four boundaries are assigned zero gradient condition for $U$ and $\alpha_{1}$ but the $p_{\text {rgh }}$ employs a Dirichlet condition equal to the operating pressure (equal to $101,325 \mathrm{~Pa}$ ). The bubbles modelled in this work are a millimeter sized bubble of radius equal to $2.5 \mathrm{~mm}$ and a sub-millimeter bubble of radius equal to $0.25 \mathrm{~mm}$. These simulations are run until $0.05 \mathrm{~s}$ and the corresponding time step constraints based on the mesh resolution are discussed in Section 4.2 and Section 4.3.

\section{Results and discussions}

In order compare the results from the dynamic and static simulations, spurious velocities, denoted by $U_{\text {sc }}$, are calculated as $\max (|\boldsymbol{U}|)$. The time averaging of an arbitrary parameter $\Phi$ and spurious velocities are represented as with an over bar as $\bar{\Phi}$ and $\bar{U}_{\text {sc }}$ respectively.

\subsection{Capillary rise}

For $2 \mathrm{D}$ capillary rise, the equilibrium height $\left(h_{\mathrm{T}}\right)$ at which when gravitational force balance the vertical component of surface tension force for a liquid column rising between two parallel plates can be theoretical calculated as

$$
h_{\mathrm{T}}=\frac{2 \sigma \cos \theta}{\Delta \rho|\boldsymbol{g}| t}
$$


where $\theta, t$, and $\Delta \rho$ are the contact angle, distance between parallel plates (equal to $1 \mathrm{~mm}$ ), and difference between densities of the phases respectively (Bullard and Garboczi, 2009). $h_{\mathrm{T}}$, based on Eq. (12), is equal to $9.91 \mathrm{~mm}$ and the capillary rise height from the simulations is calculated as

$$
h_{\mathrm{s}}=\frac{\int_{S} \alpha_{1} \mathrm{~d} S}{t}
$$

where the numerator represents the area of the liquid in the computational domain.

The capillary rise from the simulations is compared to Eq. (12) in Table 2. The temporal evolution of the capillary rise heights and spurious velocities $\left(U_{\mathrm{sc}}\right)$ are plotted in Fig. 1 and Fig. 2 respectively. Although $h_{\mathrm{S}}$ obtained from the simulations stabilize after the initial transients, the capillary rise height obtained with $C_{\text {sh }}=0.3$ oscillates slightly, by approximately $9.30 \pm 0.009 \mathrm{~mm}$, as shown in Fig. 1. This oscillation in the interface position, using $C_{\mathrm{sh}}=0.3$, also cause the periodic variation of $U_{\text {sc }}$ which is shown in Fig. 2. As the oscillations in capillary rise height are lower than $\pm 0.1 \%$ of the capillary rise height, we assume that the simulations have converged reasonably. $h_{\mathrm{S}}$ obtained when using $C_{\mathrm{sh}}=0.5$ matches the capillary rise height reported by Vachaparambil and Einarsrud (2019a) using SSF model. It is also worth pointing out that $U_{\mathrm{sc}}$ obtained using $C_{\mathrm{sh}}=0.0$ is an order larger than spurious velocities obtained with other values of sharpening coefficients, see Table 2 and Fig. 2.

\subsection{Millimeter sized stationary bubble}

The Laplace pressure in a 2D bubble can be theoretically calculated using the Young-Laplace equation as

$$
\Delta p_{\mathrm{T}}=\frac{\sigma}{R}
$$

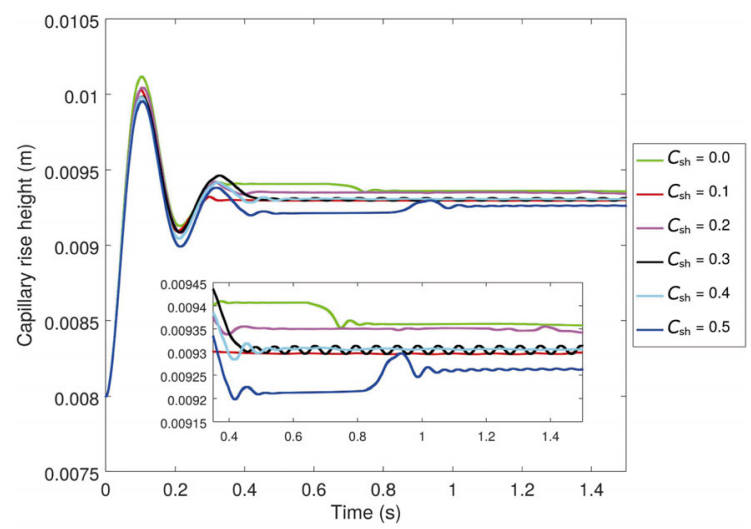

Fig. 1 Temporal evolution of capillary rise height, calculated based on Eq. (13), for various values of sharpening coefficients $\left(C_{\mathrm{sh}}\right)$.
Table 2 Variation of capillary rise height with sharpening coefficients

\begin{tabular}{cccc}
\hline$C_{\mathrm{sh}}$ & $\left.U_{\mathrm{sc}}\right|_{t=1.5 \mathrm{~s}}(\mathrm{~m} / \mathrm{s})$ & $h_{\mathrm{S}}(\mathrm{mm})$ & $E(h)=\left(h_{\mathrm{T}}-h_{\mathrm{S}}\right) / h_{\mathrm{T}}$ \\
\hline 0.0 & 0.1810 & 9.36 & 0.056 \\
0.1 & 0.0098 & 9.30 & 0.062 \\
0.2 & 0.0097 & 9.34 & 0.057 \\
0.3 & 0.0050 & 9.30 & 0.061 \\
0.4 & 0.0041 & 9.31 & 0.061 \\
0.5 & 0.0031 & 9.26 & 0.065 \\
\hline
\end{tabular}

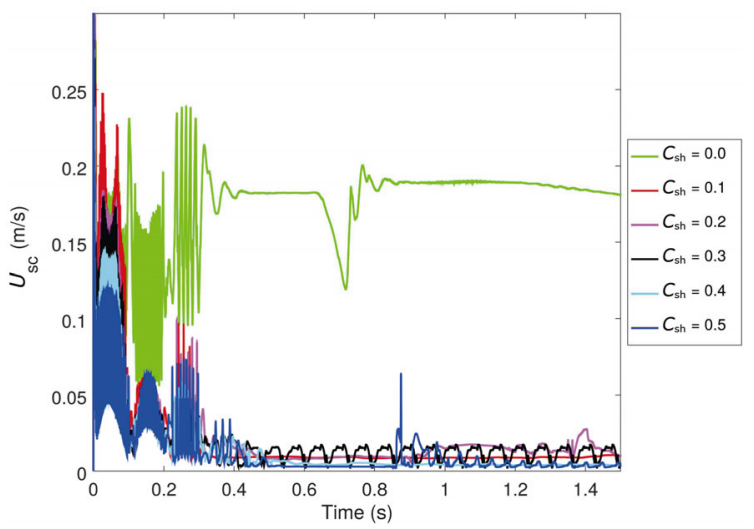

Fig. 2 Temporal evolution of spurious velocities $\left(U_{\mathrm{sc}}\right)$ during capillary rise simulations for various values of sharpening coefficients $\left(C_{\text {sh }}\right)$.

which for the bubble radius of $2.5 \mathrm{~mm}$ in the simulation is equal to $28 \mathrm{~Pa}$. For simulations, the Laplace pressure in the bubble is calculated as

$$
\Delta p_{\mathrm{S}}=\frac{\int_{V} \alpha_{2} p \mathrm{~d} V}{\int_{V} \alpha_{2} \mathrm{~d} V}-p_{0}
$$

where $p_{0}$ is the operating pressure (equal to $101,325 \mathrm{~Pa}$ ). The mesh resolution and the time step constraints (calculated based on Eq. (11)) used in the simulations are summarized in Table 3.

The stationary millimeter bubble has been modelled with the three meshes as well as for a range of sharpening coefficients between 0 and 0.5 , see Table 4 and Fig. 3 . Spurious velocities are observed on both sides of the interface for all the cases modelled, as illustrated in Fig. 3. The use of larger sharpening coefficients seems to reduce the error in calculating Laplace pressure as well as spurious velocities in the simulations, see Table 4 . Decreasing the mesh size does not always exacerbate spurious velocities which is contrast to the increasing $U_{\mathrm{sc}}$ observed with CSF model in the work by Deshpande et al. (2012) and Vachaparambil and Einarsrud (2019a). The variation between $U_{s c}$ reported in Table 4 and the work by Vachaparambil and 


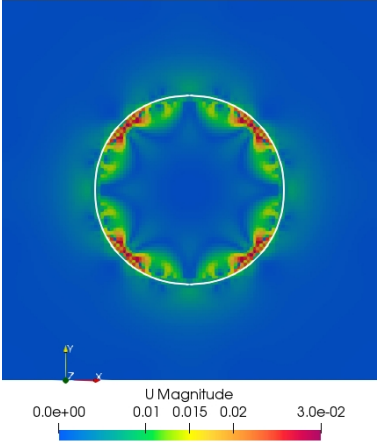

(a) $C_{\text {sh }}=0.0$

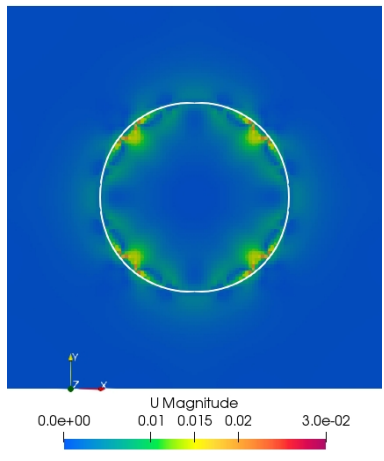

(d) $C_{\text {sh }}=0.3$

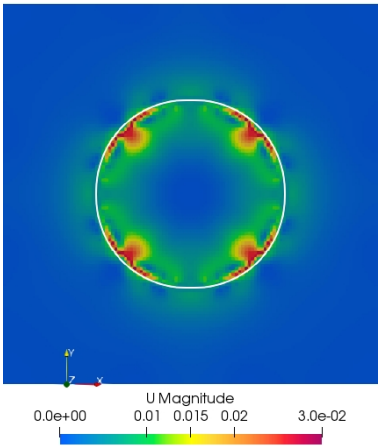

(b) $C_{\mathrm{sh}}=0.1$

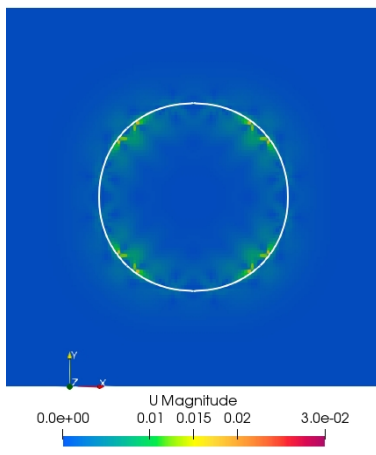

(e) $C_{\mathrm{sh}}=0.4$

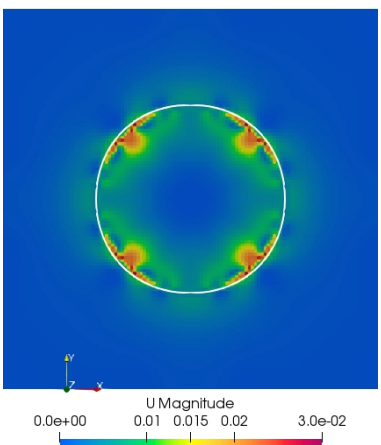

(c) $C_{\mathrm{sh}}=0.2$

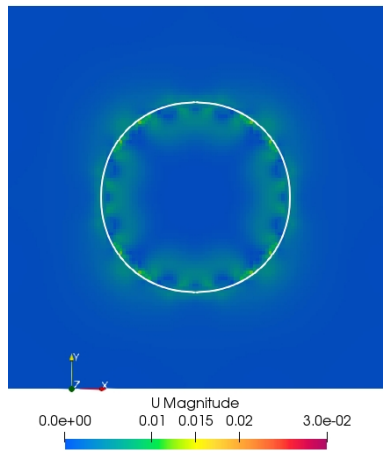

(f) $C_{\text {sh }}=0.5$

Fig. 3 Magnitude of velocity $(\mathrm{m} / \mathrm{s})$ at $t=0.05 \mathrm{~s}$ observed in the simulations of millimeter sized bubble for mesh M3 for various values of $C_{\text {sh. }}$ The white contour represents the interface at $\alpha_{1}=0.5$.

Table 3 Details of mesh and the associated maximum time step, calculated based on Eq. (11), used for stationary bubble simulations

\begin{tabular}{ccccc}
\hline Mesh & $\begin{array}{c}\text { Mesh resolution } \\
\left(\mathrm{mm}^{2}\right)\end{array}$ & $\begin{array}{c}\text { Total number } \\
\text { of cells }\end{array}$ & $2 R / \delta x^{*}$ & $\begin{array}{c}\text { Maximum time } \\
\text { step (s) }\end{array}$ \\
\hline M1 & $0.25 \times 0.25$ & 1600 & 20 & $3 \times 10^{-5}$ \\
M2 & $0.125 \times 0.125$ & 6400 & 40 & $1 \times 10^{-5}$ \\
M3 & $0.083 \times 0.083$ & 14400 & 60 & $6 \times 10^{-6}$ \\
\hline
\end{tabular}

${ }^{\star} 2 R / \delta x$ is the ratio of the bubble diameter (equal to $5 \mathrm{~mm}$ ) and the cell size.
Einarsrud (2019a) is due to the difference in the $C_{\mathrm{sh}}$ and solver setting, in Table 1, used for the simulations.

\subsection{Sub-millimeter sized stationary bubble}

A value of $2 R / \delta x$ around $50-60$ is typically used in thermal and supersaturation driven phase change processes (Samkhaniani and Ansari, 2016). Consequently, a sub-millimeter bubble, of radius equal to $0.25 \mathrm{~mm}$, is initialized in a $1 \mathrm{~mm}^{2}$ domain that is meshed by $120 \times 120$ cells and the corresponding maximum time step, calculated

Table 4 Variation of the time averaged spurious velocities $(\mathrm{m} / \mathrm{s})$, Laplace pressure $(\mathrm{Pa})$, and associated error, calculated as $E\left(\overline{\Delta p_{\mathrm{S}}}\right)=\left(\Delta p_{\mathrm{T}}-\overline{\Delta p_{\mathrm{S}}}\right) / \Delta p_{\mathrm{T}}$, with sharpening coefficients and meshes

\begin{tabular}{|c|c|c|c|c|c|c|c|c|c|}
\hline \multirow{2}{*}{$C_{\mathrm{sh}}$} & \multicolumn{3}{|c|}{ Mesh M1 } & \multicolumn{3}{|c|}{ Mesh M2 } & \multicolumn{3}{|c|}{ Mesh M3 } \\
\hline & $\bar{U}_{\mathrm{sc}}$ & $\overline{\Delta p_{\mathrm{S}}}$ & $E\left(\overline{\Delta p_{\mathrm{S}}}\right)$ & $\bar{U}_{\mathrm{sc}}$ & $\overline{\Delta p_{\mathrm{S}}}$ & $E\left(\overline{\Delta p_{\mathrm{S}}}\right)$ & $\bar{U}_{\mathrm{sc}}$ & $\overline{\Delta p_{\mathrm{S}}}$ & $E\left(\overline{\Delta p_{\mathrm{S}}}\right)$ \\
\hline 0.0 & 0.110 & 24.008 & 0.143 & 0.075 & 25.096 & 0.104 & 0.061 & 25.596 & 0.086 \\
\hline 0.1 & 0.077 & 24.173 & 0.137 & 0.051 & 25.111 & 0.103 & 0.054 & 25.651 & 0.084 \\
\hline 0.2 & 0.070 & 24.644 & 0.120 & 0.044 & 25.118 & 0.103 & 0.048 & 25.679 & 0.083 \\
\hline 0.3 & 0.065 & 24.784 & 0.115 & 0.039 & 25.123 & 0.103 & 0.042 & 25.676 & 0.083 \\
\hline 0.4 & 0.060 & 24.822 & 0.113 & 0.036 & 25.123 & 0.103 & 0.037 & 25.668 & 0.083 \\
\hline 0.5 & 0.056 & 24.845 & 0.113 & 0.034 & 25.132 & 0.102 & 0.034 & 25.700 & 0.082 \\
\hline
\end{tabular}


based on Eq. (11), is set at $0.6 \mu$ s.

The Laplace pressure in the sub-millimeter bubble, equal to $280 \mathrm{~Pa}$, is compared to the corresponding value obtained from simulations in Table 5 . The sub-millimeter bubble could be modelled with $C_{\mathrm{sh}} \leqslant 0.3$, see Fig. 4 and Fig. 5, but for sharpening coefficient of 0.4 and 0.5 the bubble numerically drifts. For $C_{\mathrm{sh}} \leqslant 0.2$, the interface undergoes slight periodic deformation which is reflected in the oscillations in $U_{\text {sc }}$ observed in Fig. 6. This deformation is not substantial enough to observe a noticeable deviation from the circular bubble shape, see Fig. 4 . At $t=0.05 \mathrm{~s}$, the simulations using $C_{\text {sh }}=0.3$ seems to have very low spurious velocities on both sides of the interface when compared to other sharpening coefficients, see Fig. 5.

\section{Conclusions}

The effect of sharpening coefficient used in Sharp Surface Force model, developed in the work by Vachaparambil and Einarsrud (2019a), is investigated for capillary rise and stationary bubbles of radii equal to 0.25 and $2.5 \mathrm{~mm}$. The solver ensures force balance between pressure gradient, surface tension, and gravitational force due to discretization

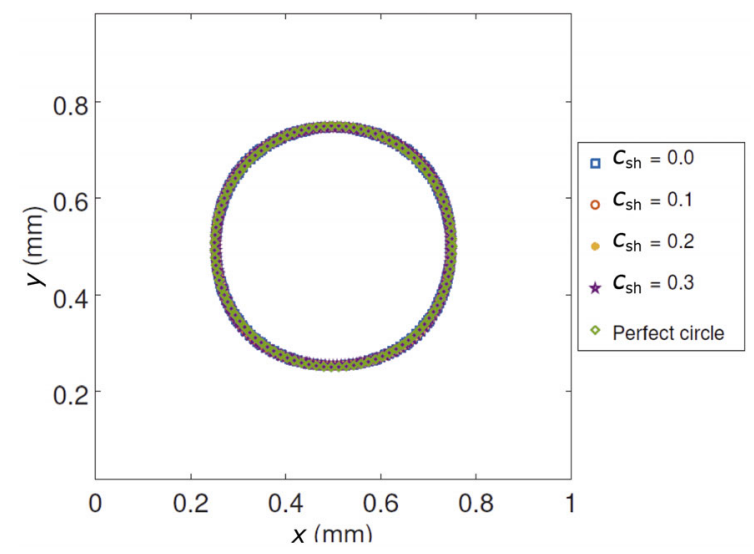

Fig. 4 Bubble morphology, represented by $\alpha_{1}=0.5$, at $t=$ $0.05 \mathrm{~s}$ for values of sharpening coefficients compared with a theoretically perfect $2 \mathrm{D}$ bubble of radius equal to $0.25 \mathrm{~mm}$.

Table 5 Time averaged spurious velocities, Laplace pressure, and associated error (calculated as $\left.E\left(\overline{\Delta p_{\mathrm{S}}}\right)=\left(\Delta p_{\mathrm{T}}-\overline{\Delta p_{\mathrm{S}}}\right) / \Delta p_{\mathrm{T}}\right)$ while modelling a sub-millimeter bubble

\begin{tabular}{cccc}
\hline$C_{\mathrm{sh}}$ & $\bar{U}_{\mathrm{sc}}(\mathrm{m} / \mathrm{s})$ & $\overline{\Delta p_{\mathrm{s}}}(\mathrm{Pa})$ & $E\left(\overline{\Delta p_{\mathrm{s}}}\right)$ \\
\hline 0.0 & 0.061 & 254.285 & 0.092 \\
0.1 & 0.039 & 254.294 & 0.092 \\
0.2 & 0.028 & 255.350 & 0.088 \\
0.3 & 0.011 & 255.279 & 0.088 \\
0.4 & Bubble numerically drifts from the original position \\
0.5 & Bubble numerically drifts from the original position \\
\hline
\end{tabular}
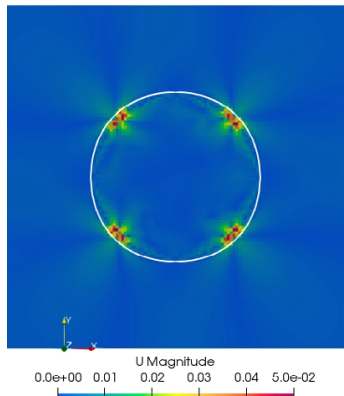

(a) $C_{\text {sh }}=0.0$

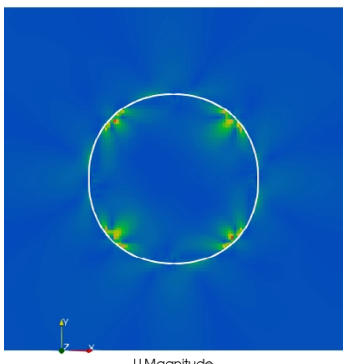

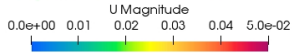

(c) $C_{\mathrm{sh}}=0.2$

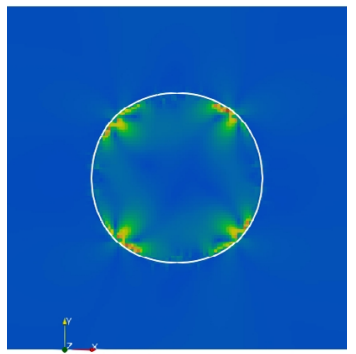

$\begin{array}{cccccc}0.0 e+00 & 0.01 & \begin{array}{c}\text { UMagnitude } \\ 0.02\end{array} & 0.03 & 0.04 & 5.00-02\end{array}$

(b) $C_{\text {sh }}=0.1$

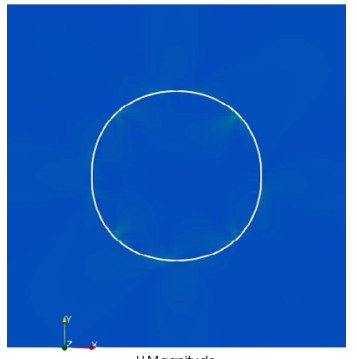

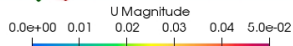

(d) $C_{\text {sh }}=0.3$
Fig. 5 Magnitude of velocity $(\mathrm{m} / \mathrm{s})$ at $t=0.05 \mathrm{~s}$ observed in the simulations of sub-millimeter sized bubble for various values of $C_{\text {sh. }}$. The white contour represents the interface at $\alpha_{1}=0.5$.

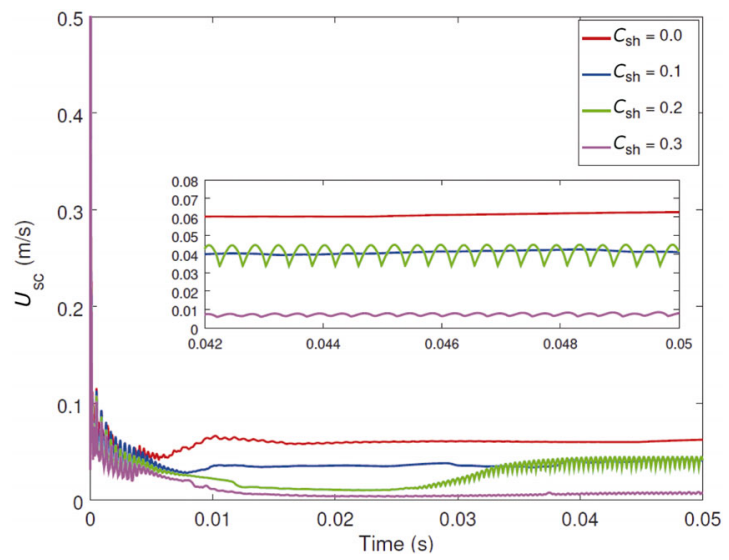

Fig. 6 Temporal evolution of spurious velocities during simulations of bubble with radius equal to $0.25 \mathrm{~mm}$ for various values of $C_{\text {sh. }}$.

and iterative procedure used to solve for $p_{\mathrm{rgh}}$ as recommended by Deshpande et al. (2012). In order to prevent the growth of spurious velocities, time step constraint based on fluid viscosity and density as well as mesh size, in Eq. (11), proposed by Deshpande et al. (2012) is used. The simulations for a range of value of sharpening coefficients, $0 \leqslant C_{\text {sh }} \leqslant 0.5$, shows that

- The use of a larger value of $C_{\text {sh }}$ generally reduces the spurious velocities in capillary rise and stationary bubble simulations.

- The mesh refinement does not always exacerbate 
spurious velocities, see Table 4, unlike while using CSF model (Deshpande et al., 2012; Vachaparambil and Einarsrud, 2019a).

- The millimeter sized bubble can be modelled with $0 \leqslant C_{\text {sh }} \leqslant 0.5$ and the three meshes. Using the finest M3 mesh and $C_{\text {sh }}$ equal to 0.5 provides the lowest spurious velocities as well as the most accurate prediction of Laplace pressure.

- The sub-millimeter bubble can be modelled with $C_{\text {sh }} \leqslant 0.3$ but the lowest spurious velocities and error in Laplace pressure are observed when $C_{\text {sh }}=0.3$.

- The capillary rise simulations show a non-linear variation of $h_{\mathrm{s}}$ with $C_{\mathrm{sh}}$ albeit the decrease in spurious velocities. The reduced spurious velocities and error in the capillary rise height is obtained when using $C_{\text {sh }}=$ 0.2 .

Although this paper investigated the effect of $C_{\text {sh }}$ for a few flow scenarios, the results show the importance of choosing an optimized value of the sharpening coefficient for future applications of SSF model to simulate two-phase flow phenomena.

\section{Acknowledgements}

This work was funded by the Department of Materials Science and Engineering at Norwegian University of Science and Technology (NTNU). The authors would also like to thank NTNU HPC and UNINETT Sigma2 for providing necessary computational resources through grant NN9741K. This paper is an extension of the poster, Vachaparambil and Einarsrud (2019b), which was presented at the 17th Multiphase Flow Conference \& Short Course and subsequently invited for publication at this journal.

\section{Funding note}

Open access funding provided by NTNU Norwegian University of Science and Technology (incl St. Olavs Hospital - Trondheim University Hospital).

\section{References}

Brackbill, J. U., Kothe, D. B., Zemach, C. 1992. A continuum method for modeling surface tension. J Comput Phys, 100: 335-354.

Bullard, J. W., Garboczi, E. J. 2009. Capillary rise between planar surfaces. Phys Rev E, 79: 011604.

Cifani, P., Michalek, W. R., Priems, G. J. M., Kuerten, J. G. M., van der Geld, C. W. M., Geurts, B. J. 2016. A comparison between the surface compression method and an interface reconstruction method for the VOF approach. Comput Fluids, 136: 421-435.

Deshpande, S. S., Anumolu, L., Trujillo, M. F. 2012. Evaluating the performance of the two-phase flow solver interFoam. Comput
Sci Disc, 5: 014016.

Greenshields, C. J. 2019. OpenFOAM User Guide version 7. OpenFOAM Foundation Ltd.

Maes, J., Soulaine, C. 2018. A new compressive scheme to simulate species transfer across fluid interfaces using the Volume-OfFluid method. Chem Eng Sci, 190: 405-418.

Pavuluri, S., Maes, J., Doster, F. 2018. Spontaneous imbibition in a microchannel: Analytical solution and assessment of volume of fluid formulations. Microfluid Nanofluid, 22: 90.

Popinet, S. 2018. Numerical models of surface tension. Ann Rev Fluid Mech, 50: 49-75.

Raeini, A. Q., Blunt, M. J., Bijeljic, B. 2012. Modelling two-phase flow in porous media at the pore scale using the volume-of-fluid method. J Comput Phys, 231: 5653-5668.

Samkhaniani, N., Ansari, M. 2016. Numerical simulation of bubble condensation using CF-VOF. Prog Nucl Energy, 89: 120-131.

Saufi, A., Frassoldati, A., Faravelli, T., Cuoci, A. 2019. DropletSMOKE++: A comprehensive multiphase CFD framework for the evaporation of multidimensional fuel droplets. Int $J$ Heat Mass Trans, 131: 836-853.

Soh, G. Y., Yeoh, G. H., Timchenko, V. 2016. Numerical investigation on the velocity fields during droplet formation in a microuidic T-junction. Chem Eng Sci, 139: 99-108.

Vachaparambil, K. J., Einarsrud, K. E. 2019a. Comparison of surface tension models for the volume of fluid method. Processes, 7: 542.

Vachaparambil, K. J., Einarsrud, K. E. 2019b. Spurious velocities in the volume of fluid method: Modeling a sub-millimeter bubble. In: Proceedings of the 17th Multiphase Flow Conference \& Short Course.

Vachaparambil, K. J., Einarsrud, K. E. 2020. Numerical simulation of bubble growth in a supersaturated solution. Appl Math Model, 81: 690-710.

Yamamoto, T., Okano, Y., Dost, S. 2017. Validation of the S-CLSVOF method with the density-scaled balanced continuum surface force model in multiphase systems coupled with thermocapillary flows. Int J Numer Method Fluids, 83: 223-244.

Open Access This article is licensed under a Creative Commons Attribution 4.0 International License, which permits use, sharing, adaptation, distribution and reproduction in any medium or format, as long as you give appropriate credit to the original author(s) and the source, provide a link to the Creative Commons licence, and indicate if changes were made.

The images or other third party material in this article are included in the article's Creative Commons licence, unless indicated otherwise in a credit line to the material. If material is not included in the article's Creative Commons licence and your intended use is not permitted by statutory regulation or exceeds the permitted use, you will need to obtain permission directly from the copyright holder.

To view a copy of this licence, visit http://creativecommons.org/ licenses/by/4.0/. 